\title{
Myringoplasty and the factors that affect its success - a case based review
}

\author{
Natalia Kyrtata, Shadaba Ahmed FRCS, ORL-HNS
}

\begin{abstract}
Myringoplasty is the surgical treatment for the repair of tympanic membrane (TM) perforations. It is indicated for treatment of recurrent ear discharge, improving conductive hearing loss, and preventing future infections, especially in high risk groups. In this case-based review, the normal anatomy and physiology of the ear is described, followed by the way conductive and sensorineural hearing loss are differentiated through a detailed history, examination and investigations, including audiometry and tympanometry. The anatomy of the TM is explored and the causes, presentation and diagnosis of TM perforations, which are a cause of conductice hearing loss, are described.
\end{abstract}

This review focuses on how myringoplasty is used to repair TM perforations, and what factors affect its success, relating the findings to four patients who have undergone myringoplasty. The underlay technique is used more frequently, yielding better results with fewer side-effects, especially for posterior perforations, whereas overlay is shown to be better for anterior perforations due to a better view of the TM, but has higher risks for graft lateralisation and blunting. Over-underlay is similar to underlay in success, but can only be applied where the handle of malleus is intact.

Temporalis fascia is the most commonly used graft for all types of perforations, although cartilage perichondrium is better for large perforations, and fat grafts are more costeffective and successful for small, central perforations. Synthetic grafts are suitable for some cases and the development of new synthetic grafts has the potential to significantly improve myringoplasty outcomes.

TM repair was shown to be independent of age, sex and timing of antibiotic administration, although chronic perforations take longer to heal. The effect of size on the surgical outcome is debatable, with different studies showing opposite results. Side-effects, alternative treatment options and potential biases in the studies are discussed, as well as the limitations of the information obtained from the cases.

The conclusion is that there is no "one-fits-all" procedure or graft that is suitable for the repair of all TM perforations, and each patient should be assessed on an individual basis, taking into consideration their own needs and wishes.

\section{NORMAL MECHANISMS - ANATOMY AND PHYSIOLOGY}

\section{Anatomy of the ear}

The ear is divided into three parts: the external, middle and inner ear (Figure 1). The external ear contains the auricle and auditory canal (external auditory meatus) which project sound waves to the tympanic membrane (TM), a thin membrane which separates the external and middle ear. The outer third of the external ear contains hair, sebaceous and ceruminous glands, where cerumen (earwax) may collect, the walls of the remaining part are bony. ${ }^{1,2}$

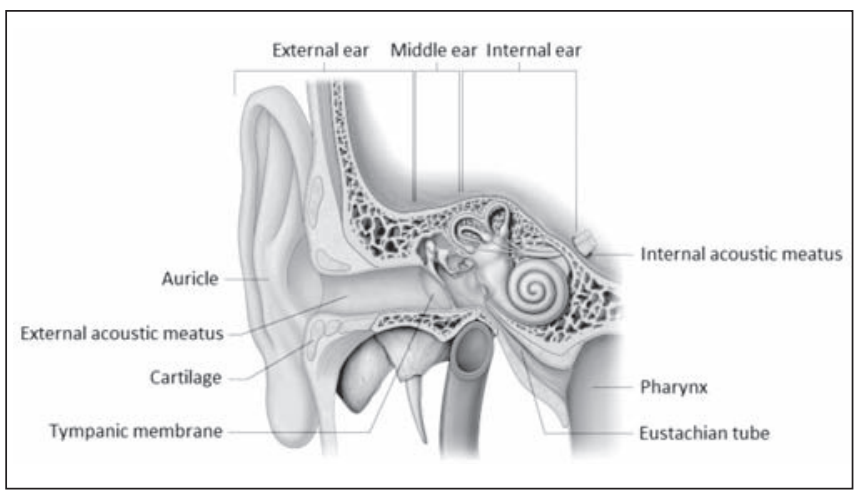

Figure 1: The anatomy of the ear ${ }^{3}$

The middle ear is an airfilled space which contains the three smallest bones of the human body, called auditory ossicles - the malleus, incus and stapes. The middle ear and nasopharynx are connected by the Eustachian tube, which is normally closed only opening during swallowing and yawning. This allows the pressure of the middle ear to equalise with the atmospheric pressure (Figure 2). Pathogens can travel from the nose and throat through the Eustachian tube into the middle ear, causing otitis media, the most common ear infection., ${ }^{4,5}$

The inner ear is functionally divided into the cochlear (the auditory system) and the vestibular system, which are responsible for hearing and balance respectively. The cochlear contains perilymph, a liquid similar in composition to cerebrospinal fluid, which covers the receptor hair cells responsible for hearing. ${ }^{1,2}$

\section{BASIC PHYSIOLOGY OF HEARING}

The auricle projects sound waves through the auditory canal to the TM, causing it to vibrate. These vibrations are amplified by the middle ear and are transmitted through the ossicles to the fluid-filled cochlea - this sets the receptor hair cells in motion, transducing the mechanical vibrations into electrical signals. Nerve impulses are generated and travel from the cochlear nucleus to the superior olivary nuclei in the pons, eventually reaching the primary auditory area of the cerebral cortex, where sound is processed (Figure 3). 
This transmission of sound through the ear is known as the air conduction (AC) system. Sound waves also cause vibrations in the skull, which are transmitted to the bony case of the inner ear, causing movement in the cochlear fluid, stimulating the receptor hair cells - this is the bone conduction (BC) system. BC is weaker than the AC since it bypasses the middle ear which serves to amplify sound. ${ }^{6}$

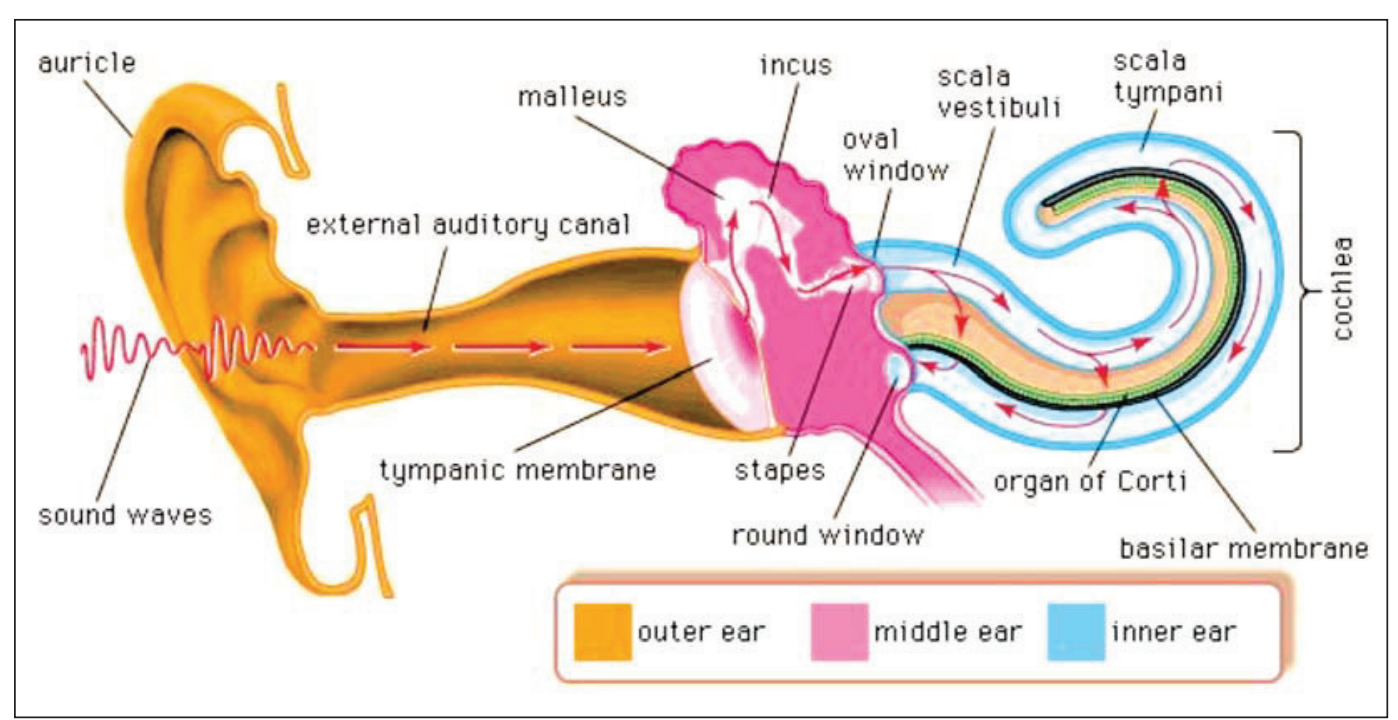

Figure 3: Hearing mechanism ${ }^{6}$

\section{Types of Hearing Loss}

Hearing loss can be grouped into two main categories: conductive hearing loss, where sound waves are not conducted effectively due to pathology in the external or middle ear, and sensorineural hearing loss, where there is damage to the inner ear (cochlear), vestibulocochlear nerve or nerve pathways leading to the brain. The term "mixed hearing loss" is used when the two occur in combination ${ }^{7}$ (see Table 1 for causes of each type).

The different types can be differentiated through a detailed history (trigger, onset and progression of hearing loss, risk factors, family history etc.), examination (otoscopy and special tests) and further investigations (e.g. audiometry).

Otoscopy can reveal blockages of the auditory canal preventing sound from reaching the TM, or a perforation in the TM, which would prevent it from vibrating effectively. Rinne's and Weber's tests are specific for determining the type of hearing loss and are always performed together

(Figure 4). In the former, a vibrating tuning fork is placed on the patient's mastoid process, testing $\mathrm{BC}$, and then in front of their auricle, testing $\mathrm{AC}$ - the latter is louder in normal hearing, and the test is positive. A negative Rinne's test occurs when $\mathrm{BC}>\mathrm{AC}$. In Weber's test, the tuning fork is placed on the patient's forehead and should be heard equally by both ears. An abnormal Weber's test occurs when it lateralises to one side (Figure 4).

Pure-tone audiometry is the most frequently performed hearing test, measuring the threshold for $\mathrm{AC}$ and $\mathrm{BC}$. The type of hearing loss is determined by the presence of an air-bone gap, the difference between the results by $\mathrm{AC}$ and $\mathrm{BC}$, which generally indicates conductive hearing loss (Figure 5). ${ }^{4}$

Tympanometry investigates pathology in the ear by testing for middle ear compliance and the mobility of the TM. Various amounts of pressure are applied in the auditory canal and a pure tone is generated, measuring and plotting the response of the TM (Figure 6). In the presence of a TM perforation, there will be an abnormally large auditory canal volume since the space of the middle ear is included in the measurements. This test should be viewed alongside pure-tone audiometry, and should not be used as a diagnostic indicator by itself.

\begin{tabular}{|l|l|}
\hline \multicolumn{1}{|c|}{ Conductive Hearing Loss } & \multicolumn{1}{c|}{ Sensorineural Hearing Loss } \\
\hline blockage (cerumen, foreign body, tumour) & age \\
fluid in middle ear (e.g. otitis media) & chronic exposure to loud noises \\
tympanic membrane perforation & genetic hearing loss \\
trauma damaging ossicles & viral infections of the inner ear, (mumps, measles) \\
tympanic membrane retraction & viral infections of auditory nerve, (mumps, rubella) \\
cholesteatoma & Ménière's disease \\
otosclerosis & acoustic neuroma \\
& meningitis \\
& encephalitis \\
& multiple sclerosis \\
& stroke \\
\hline
\end{tabular}

Table 1: Causes of conductive and sensorineural hearing loss ${ }^{5}$ 


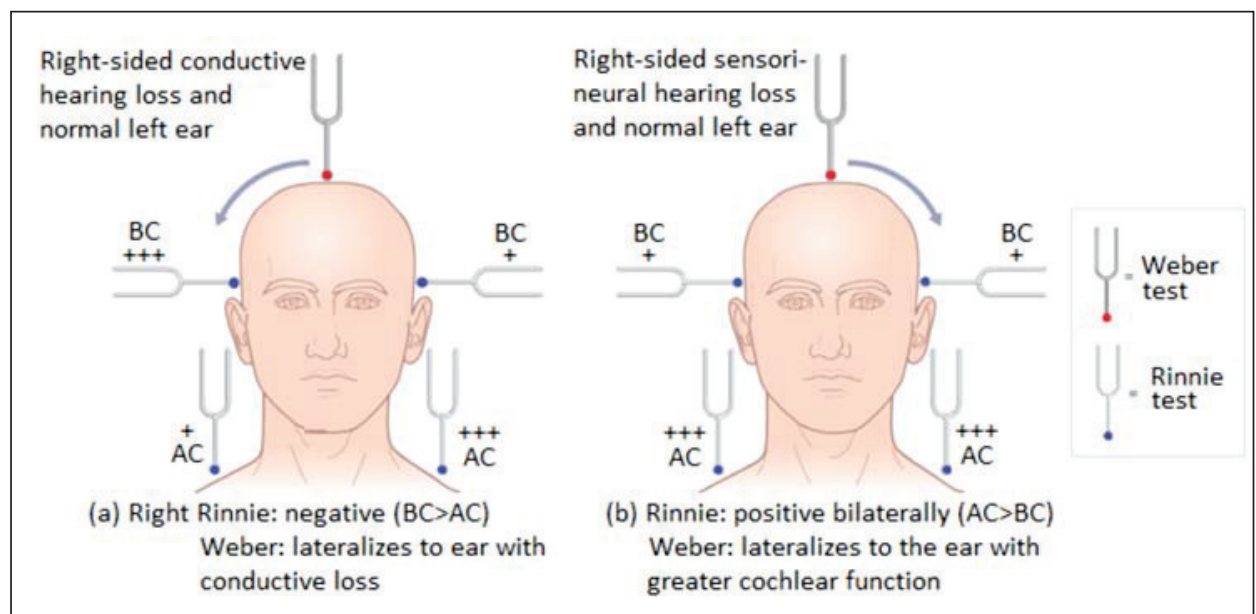

Figure 4: Tuning fork tests in (a) unilateral conductive hearing loss (b) unilateral sensori-neural hearing $\operatorname{loss}^{4}$

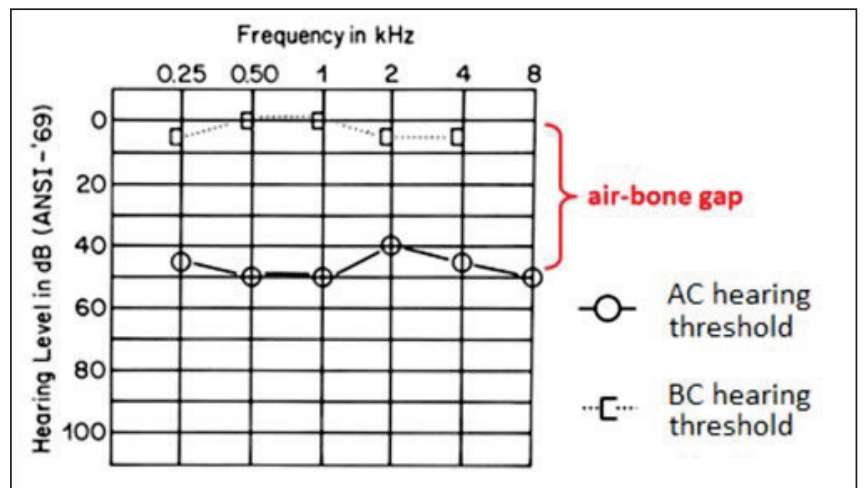

Figure 5: The patient sits in a soundproof room wearing headphones through which sounds of different volumes and frequencies are presented, first through sound attenuating headphones measuring $\mathrm{AC}$, then through a handset placed behind the auricle measuring BC. The patient presses a button each time they hear the tone and the quietest sound they can detect is recorded. ${ }^{4}$

\section{CASE HISTORIES}

Case 1 - 56 year old female

PC: hearing loss in right ear

HPC: tinnitus, feeling of fullness and reduced hearing in right ear

PMH: no history of ear infection

$\mathrm{O} / \mathrm{E}$ : large anterior central perforation in right TM, she has a pre-auricular haemangioma since birth so needs biodesign graft instead of temporalis fascia

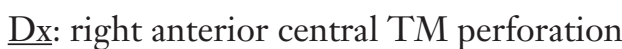

$\underline{\mathrm{Rx}}$ : right myringoplasty, per canal underlay procedure synthetic graft (biodesign),

4 weeks post-operative: graft in situ, air-bone gap closed completely, Weber's test lateralises to the right ear, facial nerve normal, but tinnitus feels louder, also has right-sided sensorineural deafness so hearing aid has been requested

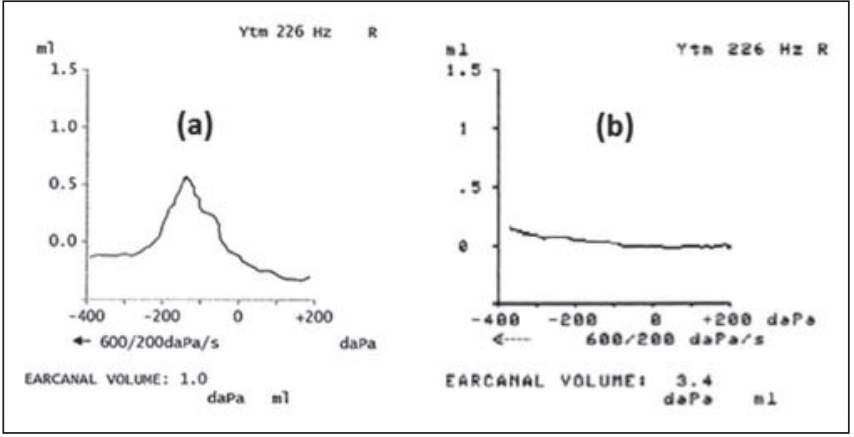

Figure 6: (a) normal tympanogram, (b) abnormal tympanogram as seen in the presence of a TM perforation ${ }^{4}$

This paper focuses on the extent to which conductive hearing loss caused by different types of TM perforations is reduced after myringoplasty, a surgical procedure used for closure of perforations. Different factors may affect the surgical outcome, and an understanding of these is necessary for both the surgeon and the patient consenting to treatment.

\section{Case 2 - 41 year old male}

PC: hearing loss in left ear after ear infection

HPC: no fever, headache, otalgia, or tinnitus but has experienced vertigo

PMH: chronic ear problems, traumatic perforation about 20 years ago, patient was told it would repair

O/E: small posterior central perforation in left TM, not infected, neurological examination was normal, normal right ear

Mild sensorineural deafness on audiogram (left)

Dx: left posterior central TM perforation

$\underline{\text { Rx}}$ : permeatal synthetic graft myringoplasty (biodesign)

4 weeks post-operative: patient is already able to hear better 
Case 3-67 year old female

PC: recurrent right ear infections

HPC: recurrent right ear infections with discharge and pain, severe hearing loss from right ear

$\underline{\text { PMH: }}$ chronic ear problems, perforated her TM aged 12 after an episode of otitis media, grommet fitted 12 years ago, improved symptoms slightly

O/E: large central perforation in right TM

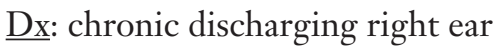

Rx: right myringoplasty - an ossiculoplasty was planned but the middle ear was full of thick mucus secretions and handle of malleus was stuck to promontory, so myringoplasty was performed

4 weeks post-operative: pack removed, graft intact

6 weeks post-operative: patient denies any hearing improvement, post-operative audiogram has not been performed yet, still gets discharging from ear and slight discomfort
Case 4 - 39 year old female

PC: left ear infection

HPC: ear gets infected when it becomes wet, no discharge or pain from her ear, no dizziness

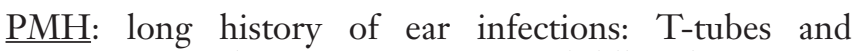
grommets fitted twice, tinnitus since childhood

O/E: right ear: TM intact, but tympanosclerosis present, left ear: large anterior perforation, dry and not infected

Dx: left otitis media

Rx: left myringoplasty, graft: temporalis fascia

2 weeks post-operative: no problems, left ear pack removed, graft in situ, wound is healthy and sutures were removed, facial nerve normal

6 months post-operative: left ear fully healed, ear has settled completely, TM was slightly retracted, patient experiences some dizziness and is being investigated by a neurologist
PC: presenting complaint

HPC: history of presenting complaint
PMH: past medical history

$\mathrm{O} / \mathrm{E}$ : on examination
Dx: diagnosis

Rx: treatment plan

Table 2: History of the four patients requiring myringoplasty


Figure 7: Case 1 Audigrams 
Right

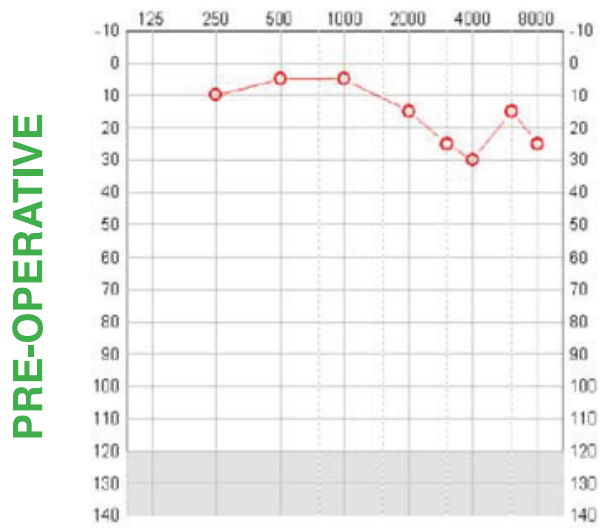

Left



Figure 8: Case 2 Audigram (post-operative not available)

\section{Right}

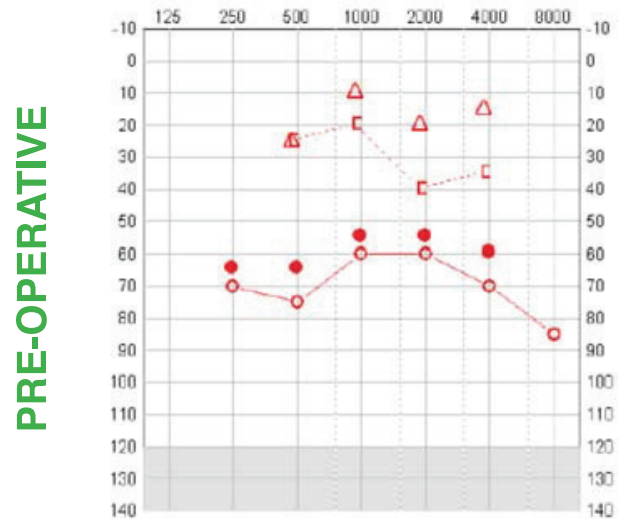

Left

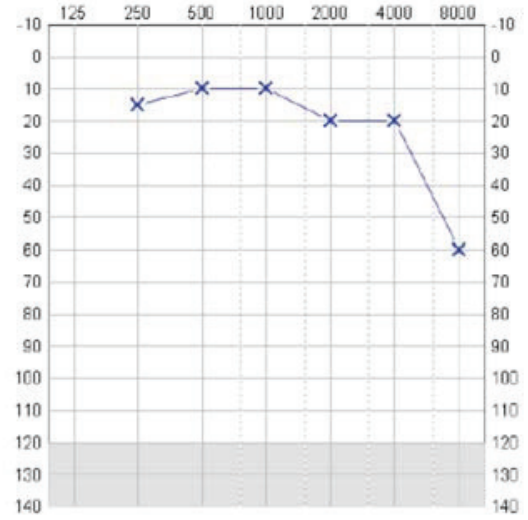

Figure 9: Case 3 Audigram (post-operative not available)

Right

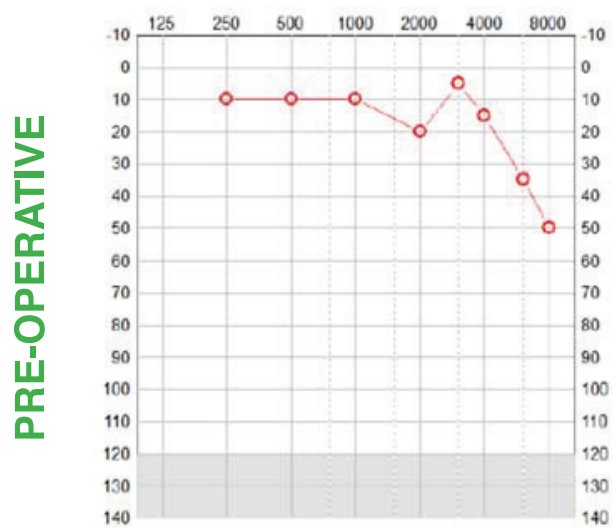

Left

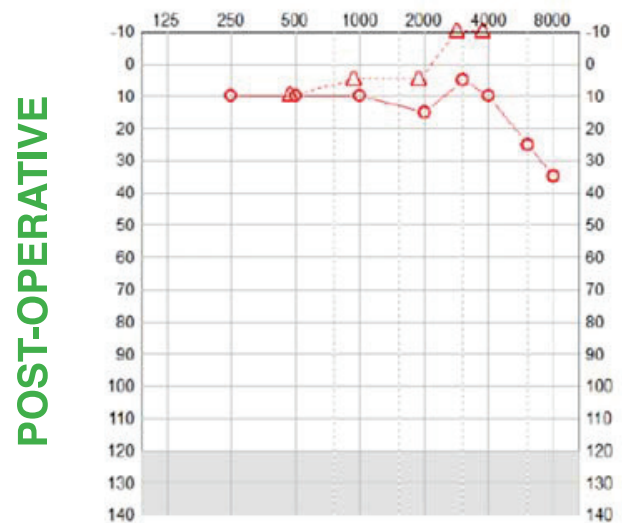

Figure 10: Case $4 \stackrel{140}{\text { Audigrams }}$
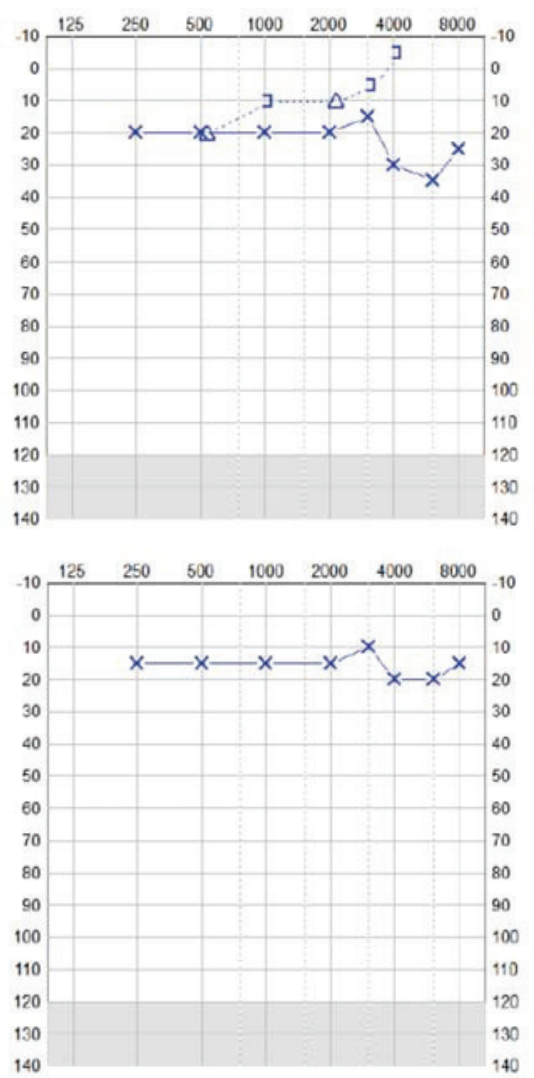


\section{Method}

The literature search was carried out through PubMed search engine. Key words including "myringoplasty," "tympanic membrane perforations" and "grafting" were used. Filters narrowed down the search results. See appendix 1 for more details. Five articles were referenced through other papers. Verbal consent by telephone was gained from four patients (Table 2) who had undergone myringoplasty.

\section{TM anatomy}

The TM is a thin membrane which separates the external ear from the middle ear. Its shape is concave, creating a "light reflex" when light reflects off it (Figure 11). The part of the TM covering the lower section of the middle ear is the "pars tensa," which, as the name suggests, is tenser than the "pars flaccida" which covers the upper section of the TM. This is because the pars tensa contains an extra layer of radially and circularly arranged connective tissue between the outer epithelial layer and inner mucosal layer, making it more robust. ${ }^{8}$
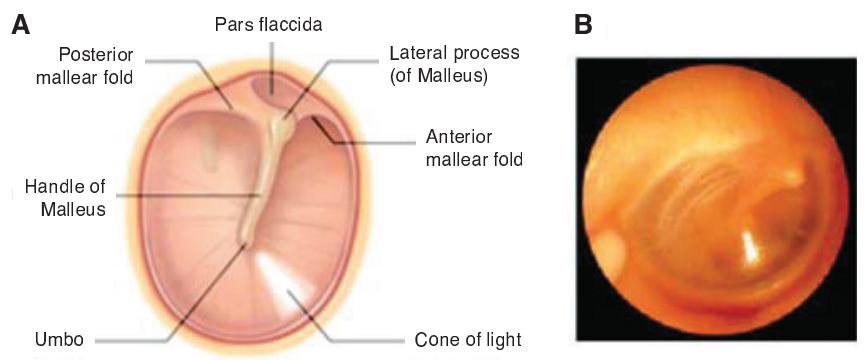

Figure 11: Tympanic membrane A. Diagram, ${ }^{3}$ B. Otoscopic view ${ }^{4}$

\section{TM perforations - causes, presentation and diagnosis}

The most common cause for TM perforations is infection, seen in Cases 3 and 4, but it can also be caused by trauma (Case 2), high-level impulsive sound pressure, or poor medical care. ${ }^{9}$ The effect on hearing depends on the size, site and shape of the perforation, as well as any associated medical conditions. They are usually diagnosed by the patient's history and through a careful examination of the ear. ${ }^{10}$

In the case of a perforation caused by middle ear infection (otitis media), the patient has symptoms of otalgia, fever and reduced hearing in one ear after having suffered from a cold. This typical picture is seen in Case 3. Pathogens travel up to the middle ear through the Eustachian tube which may block due to inflammation. Fluid accumulates in the middle ear, causing the TM to bulge. The light reflex is distorted since the TM has lost its concavity and the area may look erythematous (Figure 13)., 11 The fluid build-up may cause the TM to perforate and discharge, relieving the pain but leaving the patient with a degree of conductive hearing loss. Most perforations repair spontaneously, ${ }^{12}$ but if they do not, the exposed middle ear is at risk of further infections, ${ }^{10}$ as seen in Cases 2 and 3. These patients perforated their TM at a young age but their TM did not repair by itself. Children are at higher risk due to their narrower Eustachian tube. ${ }^{13}$

On examination, the perforation may be evident through an otoscope. The ear may need to be cleaned from cerumen to have a clearer view of the TM. ${ }^{11}$ The findings can be supported by a pure-tone audiogram to determine the extent of hearing loss. The pre-operative audiograms of Cases 1, 2 and 3 show a significant air-bone gap, which is consistent with their history (Figures 7-9).

Perforations can be central, marginal or total, depending on how much of the TM is involved, and are also defined by their location - anterior/posterior, inferior/ superior (Figure 12). The size should also be documented from the initial examination. Smaller perforations, as seen in Case 2, generally cause a smaller degree of hearing loss, however it is difficult to determine this from just one patient.

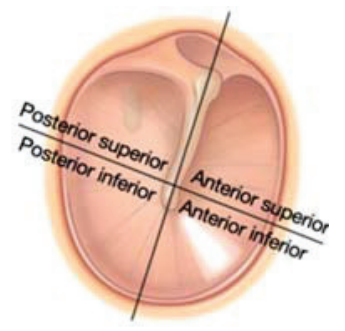

Figure 12: TM divisions ${ }^{3}$

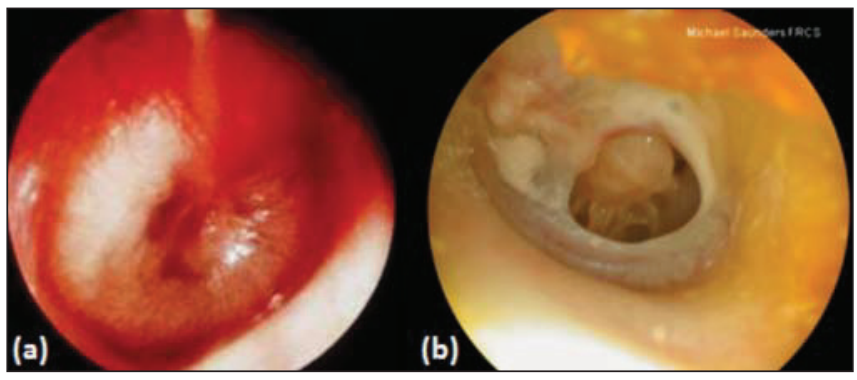

Figure 13: (a) acute otitis media (b) inferior TM perforation ${ }^{14}$

\section{Myringoplasty - background}

Until the 1950s, TM perforations were permanently covered with artificial material, but with the advancement of antimicrobials and operating microscopes, the development of tympanoplasty revolutionised ear surgery, successfully repairing the damaged TM..$^{15}$ Myringoplasty, or type 1 tympanoplasty, is the repair of a TM perforation when the auditory ossicles are normal and there is no need to examine the middle ear. The other types of tympanoplasty involve the repair of the ossicles as well as the TM (see Table 3 for indications of tympanoplasty). ${ }^{12,16}$

the size and location of the perforation does not correlate with the extent of hearing loss because of problems with the ossicular chain

the perforation is located in the anterosuperior quadrant where space is restricted

the negative pressure in the middle ear pulls the TM inwards (TM retraction)

suspected cholesteatoma

Table 3: Indications for tympanoplasty type $2-5^{16}$ 
The main indications for myringoplasty are: closure of a non-healing TM perforation to prevent recurrent ear discharge, improvement conductive hearing loss, or prevention of future infections for high risk groups such as swimmers. ${ }^{12}$ Cases 1 and 2 underwent myringoplasty to improve their hearing, whereas Cases 3 and 4 were mainly concerned about their recurrent ear infections and consequent reduced quality of life.

\section{Approach}

Depending on the type of perforation and ear anatomy of the patient, the procedure of myringoplasty may vary slightly. Patients are operated under general or local anaesthetic, although general is preferred for children. ${ }^{16}$ To access the TM, surgeons select between endaural, postaural and permeatal approach depending on their own experience and perforation site. The endaural (Figure 14a) is favourable for posterior and central perforations, whereas with a postaural incision (Figure 14b), anterior perforations are more accessible. The permeatal approach (through the ear canal) is used for small central perforations where the TM is fully visible through a speculum. The only small perforation was seen in Case 2 so a permeatal approach was suitable. In Case 1, even though the perforation was large ( $>50 \%$ of TM), the location of the patient's pre-auricular haemangioma meant that a permeatal approach was the safest option. ${ }^{12}$
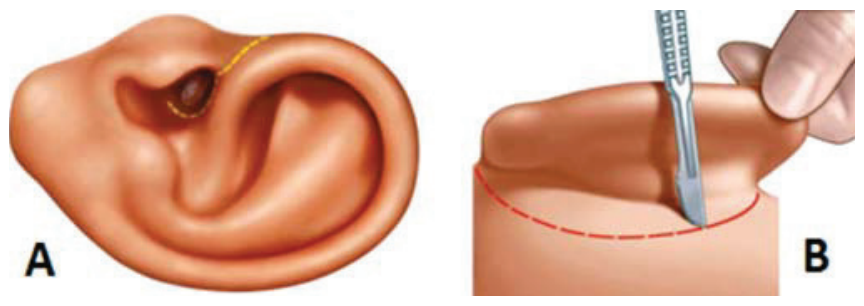

Figure 14: (a) endaural approach, (b) postaural approach ${ }^{17}$

\section{Grafts}

Local anaesthetic agent is injected at four sites around the ear, at the junction between bone and cartilage (Figure 15), as well as at the site where the graft will be taken. Different grafting materials have been used throughout the years, including veins, skin, and even paper. Those most frequently used today are perichondrium, temporalis fascia, and fat, with vein and skin grafts being rarely used due to the

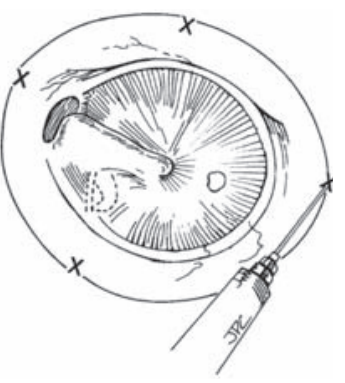

Figure 15: Injection sites for local anaesthetic higher risk of re-perforation within a few months postsurgery and the poor long-term results. ${ }^{18}$

Different grafts have a better surgical outcome depending on the type of perforation. ${ }^{19}$ Temporalis fascia seems to be the graft most commonly used for all perforation types. ${ }^{12}$ However, in a study comparing temporal fascia, tragus perichondrium, and tragus cartilage-perichondrium composite grafts, results differed between the sizes of the perforations. The study concluded that while all were suitable for repair of minor TM perforations, cartilage perichondrium composite graft showed better long-term effects, especially when used for large perforations. ${ }^{19}$ On the other hand, another study comparing fascia and cartilage grafts showed no statistical significance in the outcomes of the repair of large perforations ${ }^{20}$ Fat grafts seem be a cost-effective and suitable alternative option for small central perforations. ${ }^{21}$ Figure 16 illustrates the excision of three graft types.

Synthetic grafts have also been used in myringoplasty. ${ }^{22}$ In Case 1, the patient had a pre-auricular haemangioma since birth, making a temporalis fascia an unsuitable graft to harvest due to the abnormal vasculature of that area. In such cases, synthetic grafts are a good option as they are not harvested and do not react with the surrounding tissues. $^{23}$

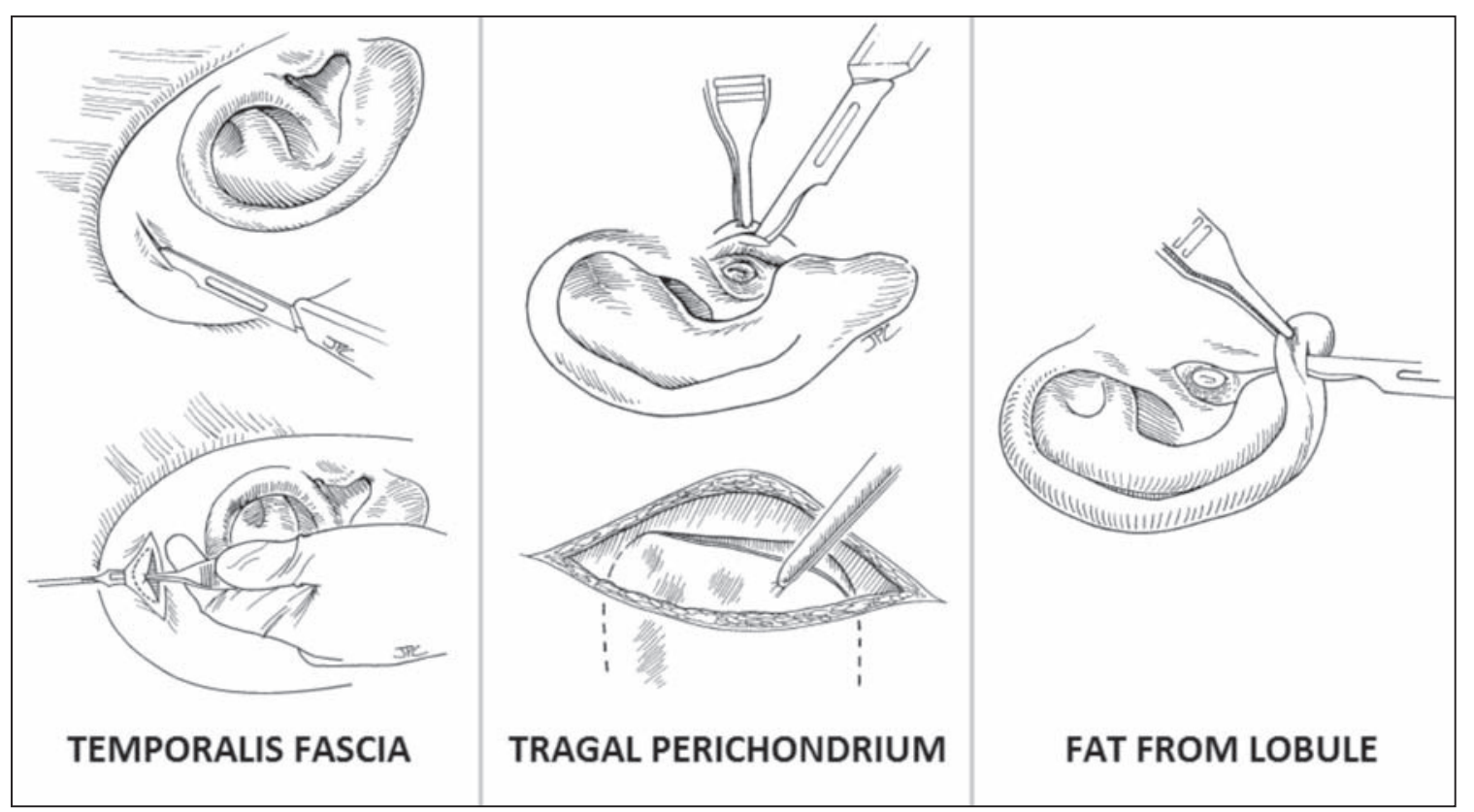

Figure 16: Excision of temporalis fascia, tragal perichondrium and lobular fat grafts $^{16}$ 


\section{Myringoplasty Technique}

Three main surgical techniques have been developed for myringoplasty: overlay, underlay and over-underlay techniques. ${ }^{24}$ The edges of the perforation are freshened so the graft can adhere to the TM. In the overlay technique, the outer epithelial layer is separated and the graft is placed lateral to the middle layer. The layers are repositioned, and the ear is packed with absorbable gelatin sponge (Spongostan) to keep the graft in place. ${ }^{16}$ In the underlay technique, the mucosal layer is separated and the graft is placed medial to the middle layer and the handle of malleus (HOM), whereas in the over-underlay technique, the TM and HOM sandwich the graft so that it lies medial to the TM but lateral to the HOM..$^{12,25}$ For every case, the edges of the graft should extend a few millimetres beyond the margins of the perforation, covering it completely. ${ }^{16}$

The underlay technique is most frequently used and has shown to be most appropriate for posterior and wellvisualised perforations. It is quick to perform, the risk of blunting and lateralisation of the graft is smaller and the TM heals in its correct anatomical position. ${ }^{24}$ However, because the graft is placed in the middle ear, the space is reduced which may increase the risk of adhesions, and it is unsuitable for certain types of perforations due to their limited visualisation..$^{24}$

Overlay has the advantage that the surgeon has a good view of the anterior portion of the auditory canal which makes repair of anterior perforations easier, however, studies have shown that healing time is longer compared to underlay, and there is a higher risk of infection. ${ }^{24,26}$ It is also associated with a higher risk of blunting and lateralisation of the graft. ${ }^{24}$

In a study examining 131 myringoplasty surgeries, the recurrence of re-perforation was $23 \%$ of patients undergoing overlay myringoplasty within a year, and $43 \%$ within two years, compared to $17 \%$ of underlay procedures in one year, and $12 \%$ after three years. ${ }^{27}$ This suggests that underlay procedures are at lower risk of re-perforation.

The over-underlay procedure has the advantage that it is placed between the HOM and the TM, so the occurrence of adhesions is less compared to the underlay technique. ${ }^{28}$ It has been suggested that it is more effective than underlay in the repair of middle to large perforations, and compared to the overlay technique, it has been shown to be easier to perform. ${ }^{29}$ However, it is only applicable in cases where the HOM is intact, so it is not always a suitable option. ${ }^{29}$

A layer of Spongostan is placed over the TM to keep everything in place, and the auditory canal is packed with antibiotic-immersed strips to prevent infection. ${ }^{16}$ Myringoplasty success appears to be independent of whether antibiotics are administered before, during, or after the operation. ${ }^{30}$

Other factors which have been investigated are the age and sex of the patients, and those with chronic perforations or perforations of unknown cause. While age and sex did not affect the surgical outcome, patients with chronic TM perforations have shown poor healing. ${ }^{30,31}$ Case 3, who had a long history of ear infections due to a perforation she acquired in childhood, proved to be the most challenging patient to treat out of the four and had the most post-operative complications.

\section{Side-effects}

Like all medical interventions, myringoplasty has a number of side-effects which the patient needs to be informed of prior to the operation. The anaesthetic risk is small but should be considered, especially in patients who have previously had reactions to anaesthetic agents. Other side-effects can be seen on Table $4 .{ }^{32}$ From those, Case 1 who had a history of tinnitus, reported that it had worsened post-operatively. This could be due to stress associated with the operation or as a side-effect from the operation itself. Case 4 experienced episodes of dizziness and Case 3 was unsatisfied with the outcomes of the operation as she does not seem to have had any improvements in her hearing. Her otalgia and otorrhoea, although reduced, have not resolved completely and she also experienced taste disturbances.

Otalgia
Discharge/bleeding from the wound site
Infection of the wound site
Reaction to the ear dressings
Reduced hearing in operated ear
Facial nerve damage
Taste disturbance
Dizziness
Tinnitus

Table 4: Side-effects of myringoplasty ${ }^{32}$

\section{Alternatives}

There are no other alternative treatment options for patients with unhealed TM perforations apart from conservative treatment with hearing aids, which some patients choose to opt for. ${ }^{32}$ Patients will still have to take preventative measures to avoid infections, such as wearing earplugs for swimming and trying to keep their ear as dry as possible.

\section{Further comments \& conclusions}

Myringoplasty is an excellent treatment option for patients suffering from the complications of a TM perforation. It is, however, unsuitable for some cases, such as those where the middle ear needs to be examined, in which case a tympanoplasty would be more appropriate.

Many studies conclude that temporalis fascia is associated with better surgical outcomes. It is also the graft most commonly used in practice. So although the studies state that the surgeons are skilled in harvesting different types of grafts, there is still the possibility that the familiarity of using temporalis fascia could introduce a bias in favour of it. In one study, a key factor which determined the success of myringoplasty was the surgical approach, suggesting that the clinical outcome is primarily attributed to technical rather than clinical factors. ${ }^{33}$

The use of synthetic materials has shown to be 
favourable in some cases where harvesting a graft is unsuitable. They can be equally effective as native tissue grafts and have the potential of shortening the operative time significantly. ${ }^{34}$ Recent research has looked into the use of silk fibroin as an alternative synthetic graft. The biological response to this material appears to be similar to other biomaterials used in today's clinical practice. ${ }^{35}$ Further research to investigate such materials in vivo could potentially introduce a more successful graft for the repair of TM perforations. ${ }^{9}$

There were some limitations with the cases used for this review. Cases 2 and 3 have not had their post-operative audiogram, which makes it hard to get an objective picture of any potential hearing improvement. Also, not all details of the surgical procedure were documented in their electronic notes, so the comparison of other study findings with these cases was not always possible.

The extent to which the size of the perforation affects the extent of hearing loss is debatable. Although different techniques have better outcomes for repairing perforations of different sizes, several studies ${ }^{31,36}$ have indicated that perforation size does not influence the healing rate, whereas others have shown that it does. ${ }^{37}$

Myringoplasty success seems to be independent of age and sex. The different sizes and sites of TM perforations have shown to be better repaired with certain approaches and techniques.

Overall, there does not seem to be one single technique that would yield the best results for all TM perforations and each case should be assessed individually to offer the most suitable treatment option for each individual patient.

\section{REFERENCES}

1. Tortora, G. J. (2011) Principles of anatomy and physiology. 13th ed. Hoboken, N.J. : Wiley.

2. Kumar, P. J. \& Clark, M. L. (2012) Kumar and Clark's Clinical Medicine 8th ed: Edinburgh: Churchill Livingstone.

3. Drake, R. L., Vogl, A. W. \& Mitchell, A. W. M. (2009). Gray's anatomy for students. 2nd ed. Philadelphia, Pa. : Churchill Livingstone.

4. Lim, E. K. S., Loke, Y. K. \& Thompson, A. M. (2007) Medicine and surgery : an integrated textbook: Edinburgh : Churchill Livingstone.

5. NHS Choices. Hearing loss - causes. [20/03/2015]; Available from: http://www.nhs.uk/Conditions/ Hearing-impairment/Pages/Causes.aspx.

6. Encyclopaedia Britannica. Transmission of sound by bone conduction. [20/03/2015]; Available from: http:// www.britannica.com/EBchecked/topic/175622/ human-ear/65049/Transmission-of-sound-by-boneconduction.

7. American Speech-Language-Hearing Association. Types of hearing loss. [March 2015]; Available from: http://www.asha.org/public/hearing/SensorineuralHearing-Loss/.

8. Clarke, R. (2014) Lecture notes: diseases of the ear, nose and throat: Chichester : Wiley-Blackwell.
9. Oktem, F., Toprak, M., Yener, M., Bozdag, E., Sunbuloglu, E. \& Toprak, T. Vibration characteristics of grafts for the tympanic membrane. Advances in Therapy. 2007;24(1):81-90. Epub 2007/05/29.

10. Howard, M. L. Middle ear, tympanic membrane, perforations. MedScape; [updated 14/07/2014 20/03/15]; Available from: http://emedicine. medscape.com/article/858684-overview.

11. Kenny, T. Perforated eardrum. Patient.co.uk; [updated 04/11/13 20/03/15]; Available from: http:// www.patient.co.uk/health/perforated-eardrum.

12. Aggarwal, R. S., Saeed, S. R. \& Green, K. J. M. Myringoplasty. The Journal of Laryngology and Otology. 2006;120(6):429-32.

13. Kenny, T. Ear infection (Otitis Media). Patient.co.uk; [updated 13/12/1220/03/15]; Available from: http:// www.patient.co.uk/health/ear-infection-otitis-media.

14. Bristol ENT Partnership. Eardrum perforations. 2010 [20/03/15]; Available from: http://www.entbristol. co.uk/otoscopy.php.

15. Kohn, F. E., Feijen, J, \& Feenstra, L. New perspectives in myringoplasty. The nternational Journal of Artificial Organs. 1984; 7(3):151-62. Epub 1984/05/01.

16. Bluestone, C. R. Surgical Atlas of Pediatric Otolaryngology 2nd ed. BC Decker; 2002.

17. ENT for medical students. 2013 [20/03/15]; Available from: http://ent4students.blogspot.co.uk/2013/05/ ent-pictures-ear.html.

18. Sarkar, S. A review on the history of tympanoplasty. Indian Journal of Otolaryngology and Head and Neck Surgery : official publication of the Association of Otolaryngologists of India. 2013;65(Suppl 3):455-60. Epub 2014/01/16.

19. Zhang, Z. G., Huang, Q. H., Zheng, Y. Q., Sun, W., Chen, Y.B. \& Si, Y. Three autologous substitutes for myringoplasty: a comparative study. Otology and Neurotology : official publication of the American Otological Society, American Neurotology Society [and] European Academy of Otology and Neurotology. 2011;32(8):1234-8. Epub 2011/09/17.

20. Yung, M., Vivekanandan, S. \& Smith, Randomized, P. study comparing fascia and cartilage grafts in myringoplasty. The Annals of Otology, Rhinology, and Laryngology. 2011;120(8):535-41. Epub 2011/09/20.

21. Ozgursoy, O. B. \& Yorulmaz, I. Fat graft myringoplasty: a cost-effective but underused procedure. The Journal of Laryngology and Otology. 2005;119(4):277-9. Epub 2005/06/14.

22. Hakuba, N., Hato, N., Okada, M., Mise, K. \& Gyo, K. Preoperative factors affecting tympanic membrane regeneration therapy using an atelocollagen and basic fibroblast growth factor. JAMA OtolaryngologyHead and Neck Surgery. 2015;141(1):60-6. Epub 2014/10/24.

23. Singh, B. J., Sengupta, A., Das, S. K., Ghosh, D. \& Basak, B. A comparative study of different graft 
materials used in myringoplasty. Indian Journal of Otolaryngology and Head and Neck Surgery : official publication of the Association of Otolaryngologists of India. 2009;61(2):131-4. Epub 2009/06/01.

24. Sergi, B., Galli, J., De Corso, E., Parrilla, C. \& Paludetti, G. Overlay versus underlay myringoplasty: report of outcomes considering closure of perforation and hearing function. Acta Otorhinolaryngologica Italica : organo ufficiale della Societa italiana di otorinolaringologia e chirurgia cervico-facciale. 2011;31(6):366-71. Epub 2012/02/11.

25. Rogha, M., Berjis, N., Taherinia, A. \& Eshaghian, A. Comparison of tympanic membrane grafting medial or lateral to malleus handle. Advanced Biomedical rResearch. 2014;3:56. Epub 2014/03/15.

26. Singh, M., Rai, A., Bandyopadhyay, S. \& Gupta, S. C. Comparative study of the underlay and overlay techniques of myringoplasty in large and subtotal perforations of the tympanic membrane. The Journal of Laryngology and Otology. 2003;117(6):444-8. Epub 2003/06/24.

27. Doyle, P. J., Schleuning, A. J. \& Echevarria, J. Tympanoplasty: should grafts be placed medial or lateral to the tympanic membrane. The Laryngoscope. 1972;82(8):1425-30. Epub 1972/08/01.

28. She, W., Dai, Y., Chen, F., Qin, D. \&, Ding, X. [Comparative evaluation of over-under myringoplasty and underlay myringoplasty for repairing tympanic membrane perforation]. Lin chuang er bi yan hou tou jing wai ke za zhi = Journal of Clinical Otorhinolaryngology, Head, and Neck Surgery. 2008;22(10):433-5. Epub 2008/08/30.

29. Kartush, J. M., Michaelides, E. M., Becvarovski, Z. \& LaRouere, M. J. Over-under tympanoplasty. The Laryngoscope. 2002;112(5):802-7. Epub 2002/08/02.

30. Hung, T., Knight, J. R. \& Sankar, V. Anterosuperior anchoring myringoplasty technique for anterior and subtotal perforations. Clinical Otolaryngology and Allied Sciences. 2004;29(3):210-4. Epub 2004/05/15.

31. Westerberg, J., Harder, H., Magnuson, B., Westerberg, L. \& Hyden, D. Ten-year myringoplasty series: does the cause of perforation affect the success rate? The Journal of Laryngology and Otology. 2011;125(2):126-32. Epub 2010/11/17.

32. Derby Hospitals NHS Foundation Trust. Myringoplasty/Tympanoplasty - Patient information. 2010 [20/03/15]; Available from: https://www. derbyhospitals.nhs.uk/easysiteweb/getresource.axd?as setid $=2990 \&$ type $=0 \&$ servicetype $=1$.

33. Albera, R., Ferrero, V., Lacilla, M. \& Canale, A. Tympanic reperforation in myringoplasty: evaluation of prognostic factors. The Annals of Otology, Rhinology, and Laryngology. 2006;115(12):875-9. Epub 2007/01/12.

34. Vos, J. D., Latev, M. D., Labadie, R. F., Cohen, S. M., Werkhaven, J. A. \& Haynes, D. S. Use of AlloDerm in type I tympanoplasty: a comparison with native tissue grafts. The Laryngoscope. 2005;115(9):1599602. Epub 2005/09/09.

35. Levin, B., Redmond, S. L., Rajkhowa, R., Eikelboom, R. H. \& Marano, R.J. \&Atlas, M. D. Preliminary results of the application of a silk fibroin scaffold to otology. Otolaryngology-Head and Neck Surgery : official journal of American Academy of OtolaryngologyHead and Neck Surgery. 2010;142(3 Suppl 1):S33-5. Epub 2010/03/05.

36. Karela, M., Berry, S., Watkins, A. \& Phillipps, J. J. Myringoplasty: surgical outcomes and hearing improvement: is it worth performing to improve hearing? European Archives of Oto-rhino-laryngology : official journal of the European Federation of OtoRhino-Laryngological Societies (EUFOS) : affiliated with the German Society for Oto-Rhino-Laryngology - Head and Neck Surgery. 2008;265(9):1039-42. Epub 2008/01/16.

37. Lee, P., Kelly, G. \&, Mills, R. P. Myringoplasty: does the size of the perforation matter? Clinical otolaryngology and allied sciences. 2002;27(5):331-4. Epub 2002/10/18. 